CORRESPONDENCE

\title{
Expanding the spectrum of PLAG1-rearranged lipoblastomas arising in patients over 45, with identification of novel fusion partners
}

(c) The Author(s), under exclusive licence to United States \& Canadian Academy of Pathology 2021

Modern Pathology (2022) 35:283-285; https://doi.org/10.1038/s41379021-00888-6

\section{TO THE EDITOR:}

Recently, Fritchie et al. published 22 cases of lipoblastomas presenting in older children and adults (age range 4-44 years, median 10 years) and identified PLAG1 fusion with six novel fusion partners in $59 \%$ of their cohort ${ }^{1}$. As detailed in their publication, lipoblastomas are benign neoplasms of embryonal white fat with a characteristic morphology, typically demonstrating PLAG1rearrangements. To date, different PLAG1 fusion partners have been described, including COL1A2, COL3A1, HAS2, RAD51L1, RAB2A, BOC, CHCHD7, SRSF3, HNRNPC, PCMTD1, EEF1A1, YWHAZ, CTDSP2, PP2R2A, DDX6,KLF10, and KANSL1 $L^{1-8}$. As a result, exon 2 or 3 of PLAG1 combines with the $\mathrm{N}$-terminal side of the gene partner, leading to an accelerated transcription of the fusion gene.

Lipoblastomas in patients over 3 years of age are thought to be exceedingly rare. These neoplasms can maturate and, in the later stage of disease, morphologically resemble "ordinary lipoma" or "fibrolipoma"1,9. The lack of a fatty component has been used as the distinguishing morphologic feature between a "pediatric fibromyxoid soft tissue tumor with PLAG1 fusion" and a "fibroblastic" lipoblastoma ${ }^{2}$. Usually, lipoblastomas are not analyzed by immunohistochemistry, RNA-sequencing or FISH. Therefore, the identification of a "fibrofatty" or myxoid tumor as a lipoblastoma in adults depends on the awareness that this tumor entity also occurs in an advanced age group. In addition, immunohistochemical staining with CD34, PLAG1, and focally desmin and the presence of a PLAG1-rearrangement facilitate the diagnosis.

Very recently, we have encountered two lipoblastomas arising in male patients 47 and 57 years of age, respectively. This correspondence aims to describe two molecularly proven lipoblastomas in individuals older than recently reported and expand the spectrum of novel PLAG1 fusion partners, namely PI15 and ZEB2.

Case 1: A 47-year old male patient presented with abdominal pain. CT (not shown) and MRI showed a $16.5 \mathrm{~cm}$ large, wellencapsulated, solid mass with heterogeneous enhancement localized in the lateral para-vesical space (Fig. 1A, B). The patient underwent resection. Grossly, the tumor was firm, yellow-tan, and lobulated. Histology showed a circumscribed lesion with a vague multinodular growth pattern (Fig. 1C, D). The tumor showed a predominant fibrous, partly myxoid stroma and variable cellularity.
Embedded in the fibrous stroma with dense fibrohyalinized background and branching vessels, bland plump oval to spindled tumor cells were seen. In addition, mature fat cells could be identified. Focal, myxoid nodules with a plexiform network of thinwalled vessels, fat cells, single lipoblasts, and primitive mesenchymal cells were found. Immunohistochemistry showed nuclear staining for PLAG1 (clone 3B7, 1:100, Novus Biologicals, Littleton, $\mathrm{CO}$ ), focal staining for desmin (clone D33, RTU; Dako), and strong staining for CD34 (clone QBEnd10, RTU; Dako, Glostrup, Denmark) in tumor cells (Fig. 1D inset). Pan-TRK (rabbit monoclonal antibody, clone EPR17341, RTU, Roche, Ventana, Tucson, AZ) showed weak cytoplasmic staining, whereas STAT6 (clone D-1, 1:100; Santa Cruz Biotechnology, Santa Cruz, CA), CDK4 (clone DCS-35, RTU, Santa Cruz Biotechnology), MDM2 (clone IF2, 1:30; Calbiochem, EMD Chemicals, San Diego, CA), CD117 (clone A4502, 1:400, Dako), DOG1 (SP31, 1:100; Thermo Fischer Scientific, Freemont, CA), SOX10 (clone SP267, RTU; Roche, Ventana), and S100P (clone 16/S100P, 1:100; BD Biosciences, Wokingham, UK) were negative.

Case 2: A 57-year-old man patient presented with a $6 \mathrm{~cm}$ thigh mass and the resection was performed. On the cut surface, the lesion was lobulated, yellow-tan, myxoid, and gelatinous. Histology showed a strikingly lobulated myxoid lesion composed of bland spindle cells with tapering nuclei and indistinct cytoplasm admixed with a delicate network of thin-walled vessels (Fig. 1E, F). The individual tumor lobules were separated by fibrous septa. In addition, a small number of lipoblasts with scalloped nuclei without significant nuclear atypia were occasionally found (Fig. $1 \mathrm{~F}$ inset left). Immunohistochemistry showed diffuse positivity for CD34 and S100 protein, and nuclear staining for PLAG1 (Fig. 1F inset right) while desmin, SMA (clone 1A4, 1:5000, Sigma Aldrich, Gillingham, UK), SOX10, MDM2, CDK4, and DDIT3 (clone 9C8; Abcam, Cambridge, UK) were negative.

Fusion gene detection was performed using next-generation sequencing-based anchored multiplex PCR technique (lon Torrent Proton using the lon PI Hi-Q Sequencing 200 kit, Thermo Fischer, Waltham, MA). Analysis was performed with Archer FusionPlex Sarcoma Expanded Panel to assess the rearrangements in the following genes: ALK, BCOR, BRAF, CAMTA1, CIC, CSF1, EGFR, EPC1, ERG, ESR1, EWSR1, FGFR1, FGFR2, FGFR3, FOS, FOSB, FOXO1, FUS, GLI1, HMGA2, JAZF1, MDM2, MEAF6, MET, MGEA5, MKL2, MYOD1, NC OA1, NCOA2, NR4A3, NTRK1, NTRK2, NTRK3, NUTM1, PAX3, PDGFB, PHF1, PLAG1, PRKCA, PRKCB, PRKCD, RAF1, RET, ROS1, SS18 (SYT), STAT6, TAF15, TCF12, TFE3, TFG, USP6, VGLL2, YAP1, YWHAE. In both cases, a PLAG1-rearrangement was found, and two novel not yet described fusion partners were identified, namely PI15 (8q21.13) in case 1 and ZEB (2q22.3) in case 2 (Fig. 2). 

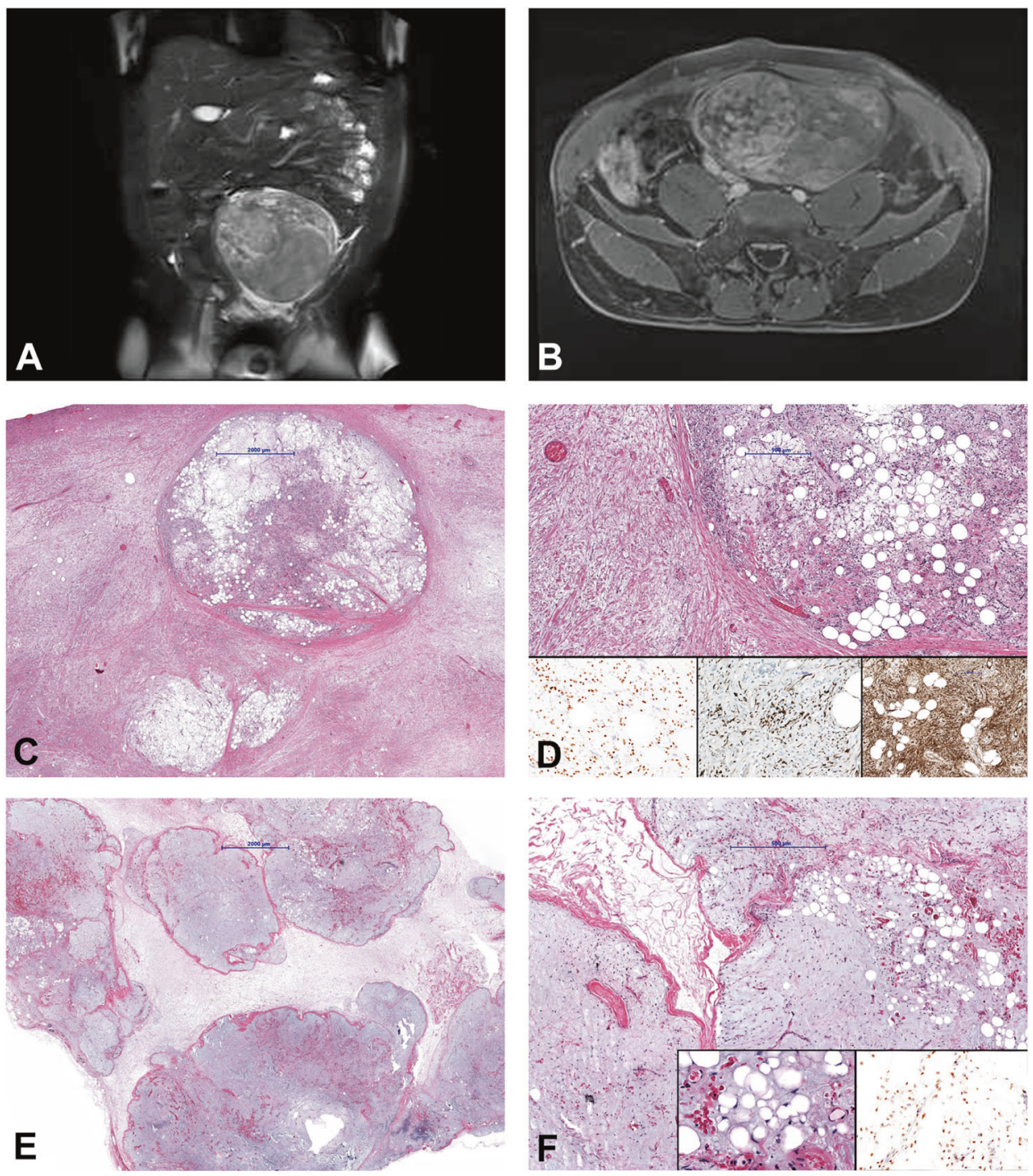

Fig. 1 Radiologic, histologic, and immunohistochemical findings. Case 1: A Coronal T2-weighted HASTE (half Fourier single-shot turbo spinecho) sequence of the pelvis shows a well-encapsulated heterogenous solid lesion with predominantly intermediate signal and hyperintense tissue streaks corresponding to myxoid tissue. B Heterogeneous enhancement of the lesion after intravenous Gadolinium application with nodular appearance and fibrous septations with sparse fatty areas. $\mathbf{C}$ The tumor is well-circumscribed with vague multinodular growth pattern with two distinct morphologies. D One part of the tumor shows a predominant fibrous component with plump oval to spindled tumor cells without cytologic atypia. Scattered mature fat cell can be seen. The other part is composed of a myxoid nodule with a plexiform network of thin-walled vessels, fat cells, single lipoblasts, and primitive mesenchymal cells (inset: PLAG1 nuclear expression in tumor cells (left), focal expression of desmin (middle), and diffuse positive staining for CD34 (right). Case 2: E Myxoid tumor with a striking lobulated growth pattern. The individual tumor lobules are separated by fibrous septa. $\mathbf{F}$ The tumor is composed of bland spindle cells with tapering nuclei and indistinct cytoplasm embedded in a myxoid stroma, admixed with a small number of lipoblasts without significant nuclear atypia (inset: lipoblasts (left) and PLAG1 nuclear expression in tumor cells (right)).

Based on morphology (fatty component present), the immunophenotype, as well as the identification of the PLAG1rearrangement in both tumors, the diagnosis of a fibroblastic lipoblastoma and a myxoid example of a lipoblastoma was made in cases 1 and 2, respectively. On their last follow-up, both patients were alive without recurrence after 4 and 2 months, respectively.

We conclude that lipoblastoma without signs of maturation and the recently described fibroblastic variant of lipoblastoma can occur in adult patients even above the age of 45 and beyond the typical locations such as extremities and trunk. Awareness of the existence of this tumor entity in adults beyond typical anatomic location expands the differential diagnoses in the group of lipomatous tumors as well as mesenchymal spindle cell tumors with CD34 expression. Immunohistochemistry and molecular analysis demonstrating PLAG1 fusion with various fusion partners facilitate the diagnosis of this rare, potentially under-recognized entity in adult patients. Additional studies and follow-up data on a larger patients' collective are needed to draw further conclusions whether the group of all reported PLAG1-rearranged tumors represents a morphologic spectrum. 

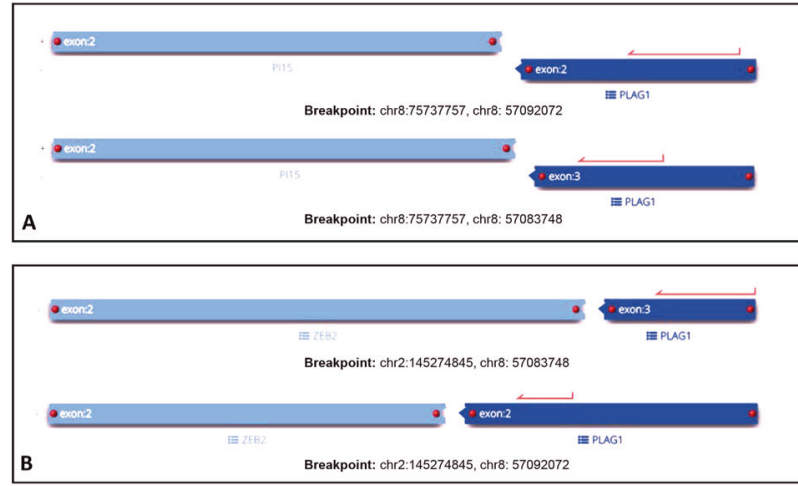

Fig. 2 Visualization of the different PLAG1 fusions detected by NGS. Visualization of A PI15-PLAG1 (case 1) and B ZEB-PLAG1 (case 2) fusions analyzed by NGS. Images show the genes and exons involved in the translocation event and the genomic location of each of the fusion.

Iva Brčić (iD) ${ }^{1}$, Jasminka Igrec ${ }^{2}$, Iris Halbwedl ${ }^{1}$, Christian Viertler $^{1}$ and Bernadette Liegl-Atzwanger (iD) ${ }^{1 凶}$ ${ }^{1}$ Diagnostic and Research Institute of Pathology, Medical University of Graz, Graz, Austria. 'Division of General Radiology, Department of Radiology, Comprehensive Cancer Centre Graz, Medical University of Graz, Graz, Austria. ${ }^{\bowtie}$ email: bernadette.lieglatzwanger@medunigraz.at

\section{REFERENCES}

1. Fritchie, K. et al. Lipoblastomas presenting in older children and adults: analysis of 22 cases with identification of novel PLAG1 fusion partners. Mod. Pathol. 34, 584-591 (2021).

2. Chung, C. T. et al. Pediatric fibromyxoid soft tissue tumor with PLAG1 fusion: a novel entity? Genes Chromosomes Cancer 60, 263-271 (2021).

3. Lopez-Nunez, O. et al. New molecular insights into the pathogenesis of lipoblastomas: clinicopathologic, immunohistochemical, and molecular analysis in pediatric cases. Hum. Pathol. 104, 30-41 (2020).
4. Hibbard, M. K. et al. PLAG1 fusion oncogenes in lipoblastoma. Cancer Res. $\mathbf{6 0}$, 4869-4872 (2000).

5. Moreno, C. et al. PLAG1-HAS2 fusion in lipoblastoma with masked $8 q$ intrachromosomal rearrangement. Cancer Genet. Cytogenet. 156, 183-184 (2005).

6. Deen, M., Ebrahim, S., Schloff, D. \& Mohamed, A. N. A novel PLAG1-RAD51L1 gene fusion resulting from a $\mathrm{t}(8 ; 14)(\mathrm{q} 12 ; \mathrm{q} 24)$ in a case of lipoblastoma. Cancer Genet. 206, 233-237 (2013).

7. Yoshida, $\mathrm{H}$. et al. Identification of COL3A1 and RAB2A as novel translocation partner genes of PLAG1 in lipoblastoma. Genes Chromosomes Cancer 53, 606-611 (2014).

8. Nitta, Y. et al. Identification of a novel BOC-PLAG1 fusion gene in a case of lipoblastoma. Biochem. Biophys. Res. Commun. 512, 49-52 (2019).

9. Shen, L. Y., Amin, S. M., Chamlin, S. L. \& Mancini, A. J. Varied presentations of pediatric lipoblastoma: case series and review of the literature. Pediatr. Dermatol. 34, 180-186 (2017).

\section{ACKNOWLEDGEMENTS}

The authors thank Prim. Dr Evelyn Gräf und Univ. Doz. Dr Johann Feichtinger for sending these interesting cases for consultation.

\section{AUTHOR CONTRIBUTIONS}

I.B.: Investigation, methodology, original draft preparation, review and editing. B.L-A.: Conceptualization, investigation, methodology, supervision, original draft preparation, review and editing. C.V. and I.H.: NGS, review and editing. J.I.: Interpretation radiologic imaging, preparing radiologic images, review and editing.

\section{COMPETING INTERESTS}

The authors declare no competing interests.

\section{ADDITIONAL INFORMATION}

Correspondence and requests for materials should be addressed to B.L-A.

Reprints and permission information is available at http://www.nature.com/reprints

Publisher's note Springer Nature remains neutral with regard to jurisdictional claims in published maps and institutional affiliations. 